\title{
Evaluation of Tensile Properties of Alcohol-Dispersed Carbon Nanotube/Low-Density Polyethylene Composites
}

\author{
Yusuf Hammed \\ Mechanical Engineering Technology \\ Federal Polytechnic Nasarawa.
}

\author{
Sanni Yusuf Yunusa \\ Mechanical Engineering Technology \\ Federal Polytechnic Nasarawa.
}

\author{
Iyahen Kenneth Osaro \\ Mechanical Engineering Technology \\ Federal Polytechnic Nasarawa.
}

\begin{abstract}
The agglomeration of carbon nanotubes makes the formation of composites difficult as it hinders uniform distribution of carbon nanotubes within the matrix. This paper investigates the effects of alcohol as a de-agglomerating chemicals i.e surfactant or dispersing agent on the carbon nanotubes. The carbon nanotubes were purified, dried and reconstituted with the dispersing agents followed by mechanical agitation using sonicator. The formed composites, after embedding the dispersed carbon nanotubes in the Low Density Poly-Ethylene (LDPE) were subjected to mechanical test. The Ultimate Tensile Strength of 4.6MPa and percentage elongation of $25.75 \%$ of $0.5 \% \mathrm{wt}$ CNT/LDPE-alcoholdispersed composites were achieved. The interfacial interaction of the carbon nanotube reinforcement in Low Density Polyethylene was studied using scanning electron microscope. The tubes were seen evenly distributed and perfectly embedded within the low density polyethylene.
\end{abstract}

Keywords: Carbon, nanotube, alcohol, dispersion, polyethylene

\section{INTRODUCTION}

Carbon nanotubes (CNT) are known to have exceptional mechanical, thermal and physical properties and this makes researchers to explore and harness it to improve the properties of polymer matrix [1].

CNT carbon atoms are covalently bonded, wrapped with graphene sheets to form seamless cylinders through $\mathrm{sp}^{2}$ hybridization with planes of bond stronger than $\mathrm{sp}^{3}$ hybridization, as in diamond. CNTs are axially strong with Young's Modulus and tensile strength in the range $270-$ $950 \mathrm{GPa}$ and $11-63 \mathrm{GPa}$ respectively [2].

CNTs possess unique electronic configuration such that single walled CNT chiral structure can be varied to be metallic or semi-conducting. Single-walled CNT exhibit current-carrying strength of about $10,000 \mathrm{~cm}^{2} \mathrm{~V}^{-1} \mathrm{~s}^{-1}$, more than silicon current-carrying strength. CNTs have 1000 times a metal's electrical conductivity. CNT has been applied as novel constituent materials in electrode for super-capacitor, fuel cell, dye-sensitized solar cell and light-emitting diode[1-2].

The electrical conductivities of disentangled multi-walledcarbon nanotube (MWCNT) are 50 times higher than the entangled MWCNTs which may be associated to straight MWCNTs having little defects [3-4].
The entangled MWCNTs have less electrical conductivity which may be as a result of the magnetic field interference in the passing of electric current whereby the field's interference can increase the current impedance [4].

The dispersion of CNT within the matrix is difficult due to interfacial energy and Van der Waal's forces within the tubes. Research has shown that with surface-active agents or surfactants which reduces the interfacial energy and increases the repulsive forces within the tubes, CNT can be unbundled and disentangled [1-3].

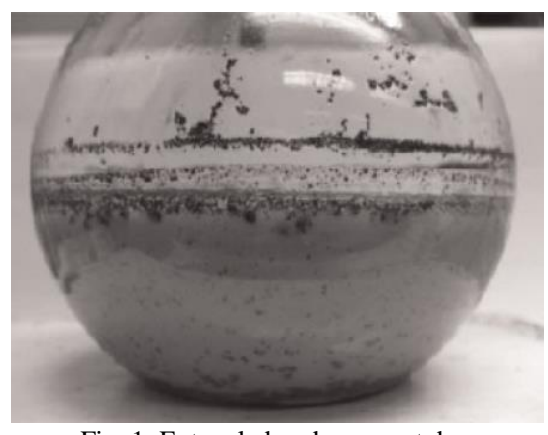

Fig. 1. Entangled carbon nanotubes

Hilding et al., 2003 [5], reported that the usual procedure of carbon nanotube dispersion is that surfactant is adsorbed on the surface of the carbon nanotube, followed by ultrasonication for minutes in order to electrostatically separate the carbon nanotubes. But recently, research has shown that surfactant is able to adhere only to the external nanotubes but the internal nanotubes remain agglomerated [6-10]. Therefore, in order to adequately unbundle the agglomerate, mechanical exfoliation prior to surface treatment of the nanotube should be done. It is on this note that ionic surfactants and non-ionic surfactants are proposed for CNT/water soluble solutions and CNT/organic solvents respectively [11-13]. Carbon nanotubes, when coated with adsorbed surfactants having high hydrophyle-lyphophyle balance can be easily dispersed in water. This is a non-covalent method which can be used for organic and in-organic dispersion of particles in aqueous solutions [12]. Sodium dodecyl sulphate and sodium dodecyl benzene sulfonic acid are known among the ionic surfactants to decrease the 
tendency of CNT agglomeration in water [14-17]. The presence of benzene ring in the surfactant is believed to make it have a better dispersive power. It has also been shown that apart from aromatic-group containing surfactants, naphthenic groups do efficiently disperse CNT [9]. An example of such is aerosol-OS (sodium disopropylnaphthalene sulfonate) which shows a high surfactant-tube affinity as revealed by spectroscopy analysis [18]. Multiwalled carbon nanotube has been efficiently dispersed in polyethylene glycol using polyethyle oxide-20- sorbitan mono-oleate, a non-ionic surfactant. This dispersion was observed to be as a result of the dispersant tail having carbon double bond [19].

\section{EXPERIMENTAL}

\subsection{Materials and methods}

As-synthesized muti-walled carbon nanotube was obtained from Centre for Genetic Engineering and Biotechnology (CGEB), Federal University of Technology Minna. Granules of transluscent low density polyethylene of $0.916 \mathrm{~g} / \mathrm{cm}^{3}$ density was purchased from Nigeria Institute of Leather and Science Technology (NILEST). Ethanol of $0.01 \mathrm{ml} \mathrm{N} / 1 \%$ acidity/alkalinity was purchased from a chemical shop in Minna, Nigeria. Compression moulding machine and the two-roll mill were obtained in NILEST, Zaria, Nigeria. TEM and SEM were carried out in South Africa.

\subsection{Purification of carbon nanotube}

Multi-walled carbon nanotubes was purified using 1:100 solid to liquid mixture of CNT and aqueous solution of $3: 1$ concentrated acetic acid and hydrochloric acid respectively. The mixture was refluxed at $70^{\circ} \mathrm{C}$ for 24 hours and continuously stirred using a magnetic stirrer at 400rpm. Then, it was cooled, the CNT was filtered and washed using distilled water and dried in oven at $60^{\circ} \mathrm{C}$ for 12 hours.

\subsection{Functionalization of carbon nanotube}

The CNTs were functionalized using 1:3 $\mathrm{HNO}_{3} / \mathrm{H}_{2} \mathrm{SO}_{4}$. The mixture was mechanically agitated on a sonicator for 18 hours. Then, the carbon nanotube was filtered and rinsed repeatedly using distilled water till $\mathrm{pH} 7$ was attained. After attaining neutrality $\mathrm{pH}$, the moist $\mathrm{CNT}$ was dried in an oven at $60^{\circ} \mathrm{C}$ for 12 hours.

\subsection{Dispersion of CNT in alcohol}

$2 \mathrm{~g}$ of functionalized carbon nanotube was added into a $3.35 \mathrm{M}$ alcohol and then sonicated for 2 hours. The dispersed solution of CNT in alcohol was filtered and oven-dried at $60^{\circ} \mathrm{C}$ for 12 hours.

\section{$2.5 \quad$ Fabrication of $C N T / L D P E$}

Two composite samples of $50 \mathrm{~g}$ low-density polyethylene with each melt-blended with $0.5 \mathrm{~g}$ and $1 \mathrm{~g}$ weight percent alcohol-dispersed CNT were prepared in a two-roll mill operating at $120^{\circ} \mathrm{C}$ and $50 \mathrm{rpm}$ for 10 minutes . The third sample was without CNT. The blended composites were compressed in a square mould at about $100^{\circ} \mathrm{C}$ in a compressing machine.
Tensile test specimens in a dog-bone shape according to ASTM-D3039 were cut from the samples and clamped in a tensometer. Upon application of force, the load-strain curve was being pen-plotted till the specimen failed and later traced for computation.

\subsection{Surface morphology}

Transmission electron microscope was used to analyse a drop of alcohol-dispersed CNT placed on TEM grids, (200 mesh) coated with carbon after the CNT-alcohol solution has been ultrasonicated for 20minutes to achieve a better image.

Scanning electron microscope (SEM, Leo Supra 55-Zeiss Inc., Germany) was used to determine the level of dispersion of the CNTs in the composites after the tensile test. The scanning was carried out at room temperature after polishing the surface of the specimen.

\section{RESULT AND DISCUSSION}

\subsection{Purification and functionalization}

Purification of carbon nanotube became necessary to avoid the interaction of unwanted impurities like amorphous carbon, residual catalyst, carbon nanoparticles that are attached to the carbon nanotube during synthesis from contaminating the process of effective dispersion of carbon nanotube in matrix.

A micro-scale inspection of the carbon nanotubes is presented in the TEM images below,: as-synthesized, purified and functionalized. The bundled size of entangled CNT is more and the unbundled size less in as-synthesized, this is expected because of its pristine nature. The bundled size is reduced in purified and further reduced in functionalized carbon nanotube but the unbundled size increased in both. The metallic impurities, amorphous and carbonaceous materials attached to the CNT have been washed away during the purification and a functional group added during the functionalization for the CNT to react with other materials [20].

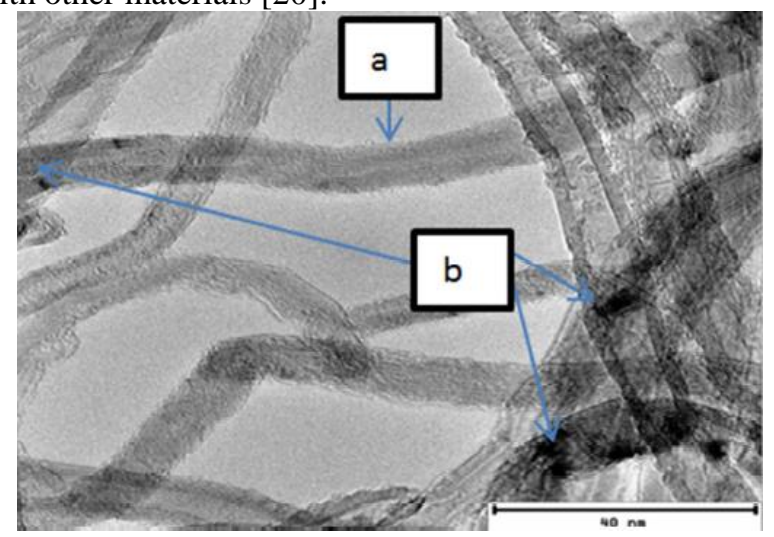

Fig. 2. TEM of as-synthesized CNT 


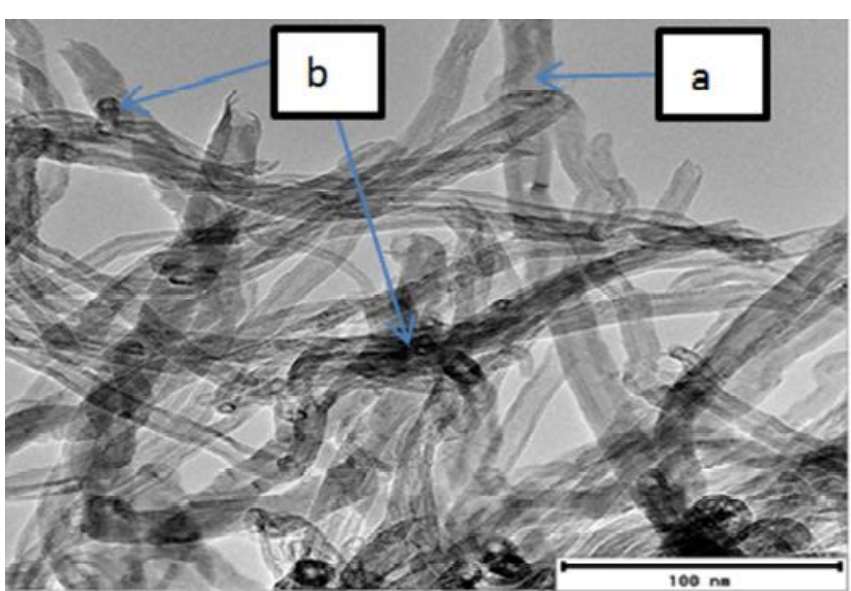

Figure 3. TEM image of purified CNT

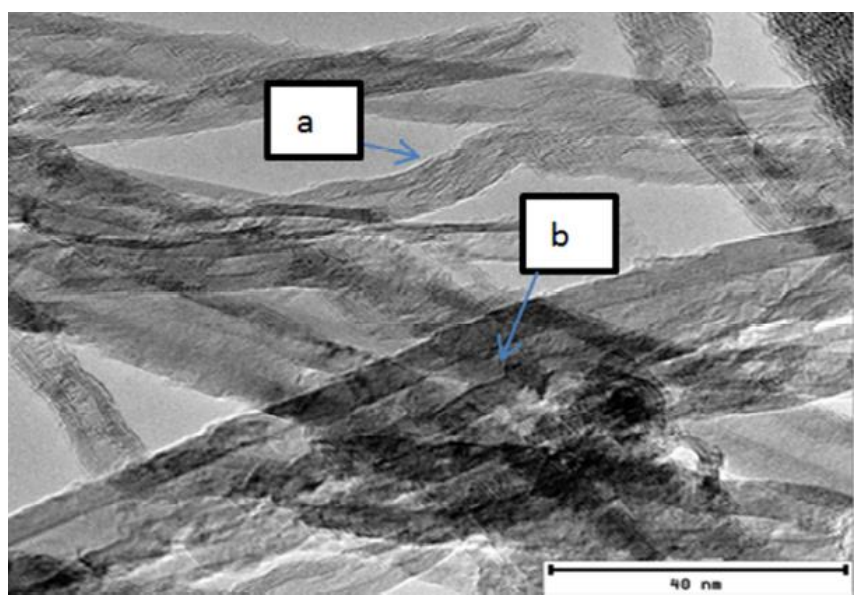

Figure 4. TEM image of functionalized CNT

\subsection{Dispersion of CNT in alcohol.}

During the sonication process of the functionalized CNT in alcohol, it was observed with bare-eye that there was a change in the colour of the solution. The longer the sonication time the darker the solution, that is, exfoliation of the carbon nanotubes in the alcohol till it was stable for the period of the two hours. The same nature of observation in the work of Dujardeen et al., 2012 [21].

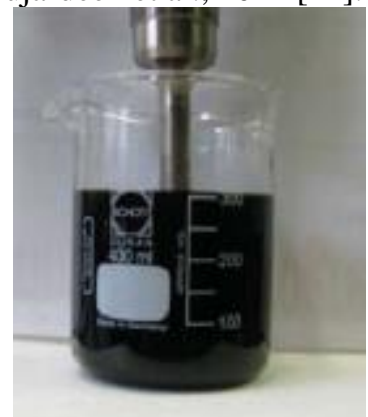

Figure 5. An exfoliated carbon nanotube after the sonication process.

The entaglement of MWNT is high as a result of the strong cohesive energy between the tubes which is in the order of $36 \mathrm{kT}[22]$.

\subsection{Tensile test}

The table below is the result of tensile test carried out on the samples

\begin{tabular}{llll}
\multicolumn{4}{c}{ TABLE I. RESULT OF TENSILE TEST } \\
\hline \% CNT & $\mathbf{0 \%}$ & $\mathbf{0 . 5 \%}$ & $\mathbf{1 \%}$ \\
\hline Width $(\mathbf{m m})$ & 9.6 & 9.65 & 9.7 \\
Thickness $(\mathbf{m m})$ & 2.85 & 2.80 & 2.8 \\
Area $\left(\mathbf{m m}^{2}\right)$ & 27.36 & 27.02 & 27.16 \\
Force (Newton) & 92.25 & 123.00 & 97.75 \\
UTS (MPa) & 3.37 & 4.55 & 3.60 \\
Strain & 0.2756 & 0.2650 & 0.2250 \\
\% Elongation & 27.56 & 26.50 & 22.50 \\
Young's & 12.23 & 17.17 & 16.00 \\
Modulus (MPa) & & & \\
\hline
\end{tabular}

3.3.1 Effect of CNTs on the Ultimate Tensile Strength of LDPE/CNT composite

The UTS value of LDPE increased to $135 \%$ and $107 \%$ when $0.5 \mathrm{wt} \%$ and $1 \mathrm{wt} \%$ alcohol-dispersed CNT were added. A similar trend is observed in the work of Nazlia et al., 2007 [23], where styrene butadiene stress value or tensile strength was increased to $121 \%$ and $170.26 \%$ upon adding $1 \mathrm{wt} \%$ and $10 \mathrm{wt} \% \mathrm{CNT}$ respectively. Xiao et al., 2006 [24], had 56\% increment in the ultimate tensile strength of ordinary LDPE when $10 \mathrm{wt} \%$ carbon nanotube was added.

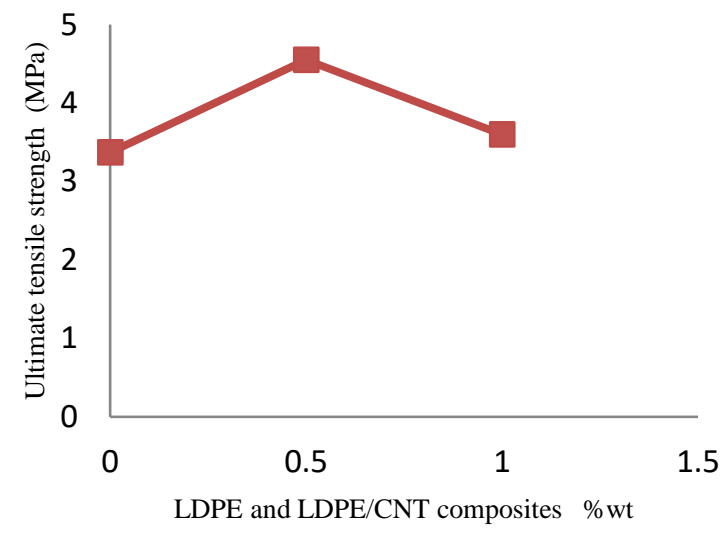

Fig. 6. Ultimate Tensile Strength of Alcohol-dispersed CNT/LDPE Composites

\subsubsection{Effect of CNTs on the Strain of LDPE/CNT composite}

Strain reduction was experienced in alcohol-dispersed CNT/composite to $96.2 \%$ and $81.64 \%$ when $0.5 \mathrm{wt} \%$ and $1 \mathrm{wt} \%$ CNT were added respectively. It agrees with the trend reported in literatures [23-24]

The more the CNT content, the lower the strain value. This may be due to the rigid nature of CNT which hinders the elongation of the composites. Also, much of the elongation is from the polymer matrix. The measured elongation is an indication that composites stiffness can be improved by CNT. 


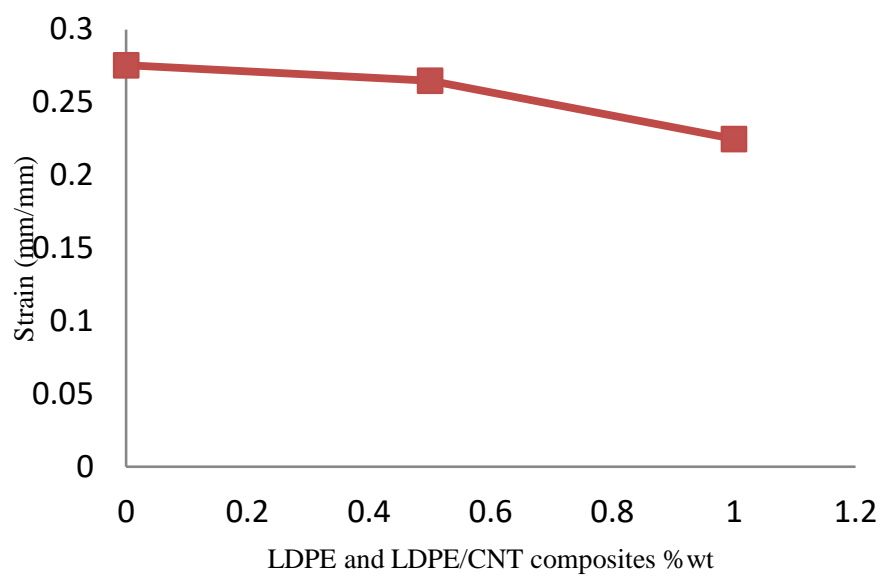

Fig. 7. Strain of Alcohol-dispersed CNT/LDPE Composites

\subsubsection{Effect of CNTs on the Young's Modulus of LDPE/CNT composite}

The Young's modulus of ordinary LDPE has increased by $40 \%$ and $31 \%$ when $0.5 \%$ and $1 \%$ alcohol-dispersed carbon nanotube were added respectively.

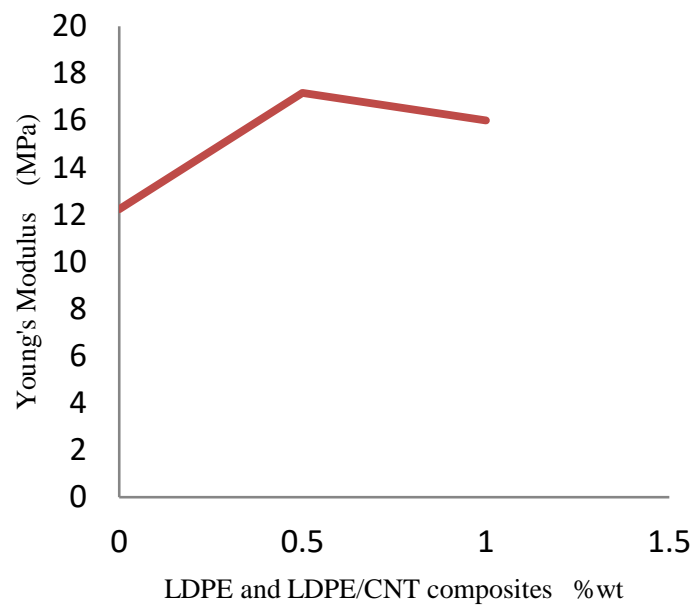

Fig. 8. Young's Modulus of alcohol-dispersed CNT/LDPE Composites

3.4 Surface Morphology of the CNT/LDPE Composites The figure below is a result of the scanning electron microscope. The result shows that there is a cordial interfacial bonding between the particles of the carbon nanotube and the LDPE as it can be observed that the tubes are effectively embedded in the LDPE without bundles of CNT randomly distributed in the image as shown. This is the effect of the two-roll mill being able to uniformly distribute the carbon nanotube within the LDPE and the efficient disentangling through various processes as outlined above.

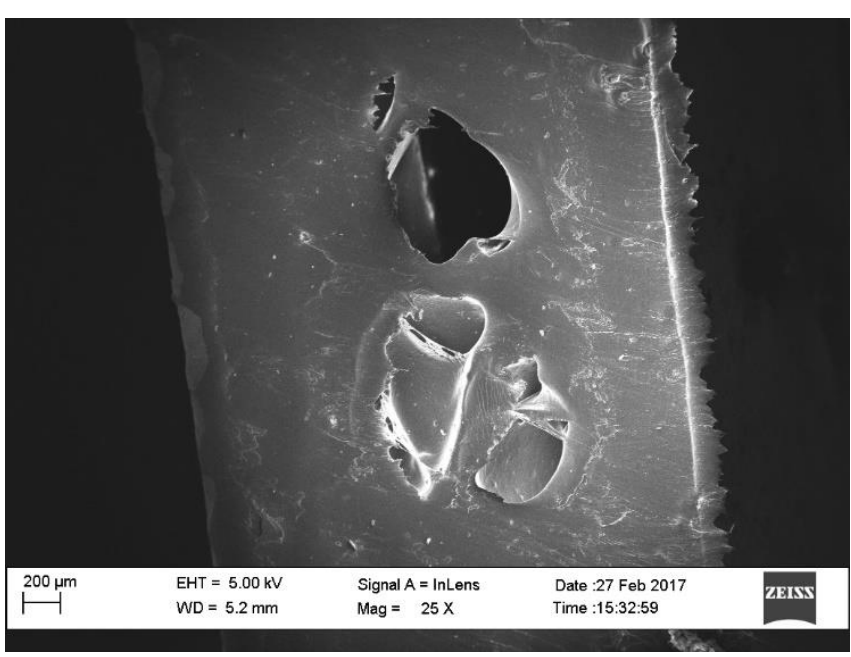

Fig. 9. SEM image CNT/LDPE composite

\section{CONCLUSION}

In summary, the effect of carbon nanotube dispersed with alcohol in low density polyethylene has been investigated. The excellent properties of carbon nanotube were harnessed to reinforce the composites. Ordinary low density poly-ethylene properties like young's modulus, ultimate tensile strength have been improved. This has proven that alcohol may also serve has a dispersing agent. The ultimate tensile strength and the young's modulus have been increased by $35 \%$ and $40 \%$ respectively Carbon Nanofiber; University Putra Malaysia (UPM)

[2] Collins, P.G. and Avouris, P. (2000) Nanotubes for electronics. Scien. Amer., 283: 62

[3] Cassell, A. M., Raymakers, J. A., Kong, J., \& Dai, H. (1999) Large Scale CVD Synthesis of Single-Walled Carbon Nanotubes. The Journal of Physical Chemistry B, 103(31), 6484-6492. doi:10.1021/jp990957s

[4] Flahaut, E., Peigney, A., Laurent, C., Marlière, C., Chastel, F., \& Rousset, A. (2000). Carbon nanotube-metal-oxide nanocomposites: Microstructure, electrical conductivity and mechanical properties. Acta Materialia, 48(14), 3803-3812.

[5] Hilding, J., Grulke, E. a., George Zhang, Z., \& Lockwood, F. (2003). Dispersion of Carbon Nanotubes in Liquids. Journal of Dispersion Science and Technology, 24(1), 1-41. doi:10.1081/DIS-120017941

[6] C. Liu and J. Choi, 'improved dispersion of carbon nanotubes in polymers at high concentrations,' Nanomaterials, vol. 2, no. 4 pp. 329-347, 2012

[7] Gong, X., Liu, J., Baskaran, S., Voise, R. D., \& Young, J. S. (2000). Surfactant-Assisted Processing of Carbon Nanotube/Polymer Composites. Chemistry of Materials, 12(4), 1049-1052. doi:10.1021/cm9906396

[8] Ajayan, P. M., \& Iijima, S. (1993). Capillarity-induced filling of carbon nanotubes. Nature, 361(6410), 333-334. doi: $10.1038 / 361333 \mathrm{a} 0$

[9] Erin L.C (2007), Dispersion and Alignment of Carbon Nanotubes in Polymer-Based Composites. Ph.D thesis: Georgia Institute of Technology.

[10] Ando, Y. (1994). The Preparation of Carbon Nanotubes. Fullerene Science and Technology, 2(2), 173- 180 doi:10.1080/15363839408009542

[11] Gong, X.; Liu, J.; Baskaran, S.; Voise, R.D., Young, J.S. Surfactant-assisted processing of carbon nanotube/polymer composites. Chem. Mater. 2000, 12 (4), 1049-1052. 
[12] Vaeck, S.V.; Merken, G.V. Some experiments on detergency in aqueous and nonaqueous media. XII. Further studies on the interaction between surfactants, electrolytes and $\mathrm{pH}$ in the deposition of hydrophobic particulate soil. Tenside Deterg. 1981, 18 (3), 126-129

[13] Dong, B., Su, Y., Liu, Y., Yuan, J., Xu, J., \& Zheng, L. (2011). Dispersion of carbon nanotubes by carbazole-tailed amphiphilic imidazolium ionic liquids in aqueous solutions. Journal of colloid and interface science, 356(1), 190-5. doi:10.1016/j.jcis.2010.12.080

[14] Breuer, O. and U. Sundararaj, (2004) Big returns from small fibers: A review of polymer/carbon nanotube composites. Polymer composites. 25(6):630

[15] Xie, X. -L., Y. -W. Mai, and X.-P. Zhou, (2005). Dispersion and alignment of carbon nanotubes in polymer matrix: A review, Materials science \& Engineering, R: Reports. R49(4):89

[16] Chae, H. G. and S. Kumar, (2006). Polymer/carbon nanotube composites - an overview. Indian Journal of Fibre \& Textile Research. 31(1):29.

[17] Fisher, F. T., R. D. Bradshaw, and L.C. Brinson (2003). Fibre waviness in nanotube-reinforced polymer composites-I: Modulus predictions using effective nanotube properties. Composites Science and Technology. 63(11):1689

[18] Rarvikar, N. R., Schadler, L. S., Vijaaraghavan, A., Zhao, Y., Wei, B., Ajayan, P. M. (2005). Synthesis and characterization of thickness-aligned carbon nanotube-polymer composite films. Chemical Materials. 17:974.

[19] Balasubramanian, K., \& Burghard, M. (2005). Chemically functionalized carbon nanotubes. Small (Weinheim an der

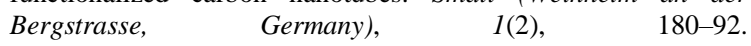
doi:10.1002/smll.200400118

[20] Bonard, J.M., Stora, T., Salvetat, J.P Maier, F., Stockli, T., Duschi, C., Forro, L., deHeer, W. A., Chatelaine, A. (1997) Purification and size-selection of carbon nanotubes. Advanced Materials. 9(10):827

[21] Dujardin, Erik, Ebbesen, T. W., Krishnan, A., \& Treacy, M. M. J. (1998). Purification of Single-Shell Nanotubes. Advanced Materials, 10(8), 611-613. doi:10.1002/(SICI)15214095(199805)10:8<611::AID-ADMA611>3.0.CO;2-8

[22] Girifalco, L. A., Miroslav, H., Lee, R.S.,(2000)Carbon nanotubes, buckyballs, ropes, and a universal graphitic potential. Physical Review B. 62(19):13104

[23] Nazlia G., Fakhrul-Razi Ahmadun, Suraya A.R. (2007). Multiwall carbon nanotubes/styrene butadiene rubber (SBR) nanocomposites. Fullerenes, Nanotubes, and Carbon Nanostructures. 15: 207-214. DOI: 10.1080/15363830701236449

[24] Xiao. K.q., Zhang L.C., Zarudi I., (2006). Mechanical and Rheological Properties of Carbon-Reinforced Polyethylene Composites. Composites Science and Technology. 67: 177-182 